\title{
Reflexiones sobre el hospital: su función en la formación de médicos
}

C. Soler-Durall

\section{Introducción}

Escribo esto desde la 'autoridad' que me otorga haber iniciado en España, en el Hospital General de Asturias, en 1960, la formación MIR, 16 años antes de que se aplicara generalizada y oficialmente en el país. La implantación del Programa de Médicos Internos y Residentes se hizo con un grupo dedicado de médicos del hospital que habían pasado por el mismo proceso y lo hicimos sin concesión burocrática alguna. Es obligado mencionar a José López-Muñiz, presidente de la Diputación y del Consejo del hospital, que hizo suyo y soportó políticamente el programa, y a Fernando Alonso Lej, director de Cirugía Torácica, que lo dirigió.

El papel del hospital en la formación de médicos no sólo es evidente, sino imprescindible. Resulta obvio también que cuanto mejor funcione un hospital, mejores resultados cabe esperar de su función pedagógica en todos los niveles de esa formación, como la iniciación médicoadministrativa -básicamente de observación, sin responsabilidades asistenciales pero de forma activa, guiada y de corta duración durante los estudios formales de Medicina- y los denominados clerkships -en el penúltimo y último año de pregraduación, controlada por los médicos residentes y la Dirección de Enfermería, y con récords escritos de actividad-. En España iniciamos esto en la Facultad Autónoma de Medicina en el Hospital de la Santa Cruz y San Pablo, con Alberto Oriol, profesor de Fisiología y jefe de estudios de la facultad, y con Adela Simón, enfermera jefe. Es el primer contacto del estudiante con el hospital, una especie de iniciación.

\section{Internados rotatorios}

Los internados rotatorios para médicos graduados son previos a la residencia. En su forma tradicional de 12 períodos de cuatro semanas ( 3 meses en medicina, 3 meses en cirugía, 1 mes en urgencias, 1 mes en obstetricia/ginecología, 1 mes en medicina familiar, 1 mes en pediatría y 2 meses electivos), y a las órdenes de los médicos residentes correspondientes, se aprende el abecé de cada situación, el funcionamiento y las posibilidades del hospital. Ningún programa de formación serio puede ni debe obviar este paso sin perjuicio de la formación como médico y como paso previo a la residencia especializada. Estas vivencias se realizan como 'visitante' en el hospital concertado con la correspondiente facultad de Medicina, o mejor - pero esto ya no lo controla la facultad, sino el matching-, en el mismo hospital en el que se realiza luego la residencia. El internado no es sustituible por prácticas al cursar las asignaturas por dos razones: porque ningún hospital ni enfermo se prestaría a ello y porque la visión global que da la graduación previa es imprescindible para entender lo que en el internado se contempla. En el internado se empieza a adquirir, además, el 'carisma' y conducta que hará respetable al profesional ante los pacientes y que madurará a lo largo de la residencia. Su supresión constituye un error muy grave.

\section{Residencia}

Son los programas de formación en las distintas especialidades, que oscilan entre 3 y 5 años,
Global Contents, SL.

Barcelona, España.

Correspondencia Dr. Carles Soler Durall. Global Contents, SL Gran Via, 534, 2. 1 . $^{\text {a }}$ E-08011 Barcelona.

E-mail

soler@globalcontents.com 
según se requieran más o menos destrezas, sólo asumibles con cierta cantidad de vivencias y con tiempo para adquirirlas.

Al final, el residente puede exhibir documentalmente y con certificados fiables lo que ha hecho y sabe hacer, su conducta humana, etc. En estos certificados se involucra toda la estructura jerárquica médica y la dirección del hospital. Ningún examen puede sustituir esto y considero también graves los intentos de intervencionismo burocrático que pueden acabar con el sistema y la calidad de la medicina, tan trabajosamente conseguida en España gracias al programa MIR.

El hospital ha de tener la capacidad de interrumpir el esfuerzo si no se dan en el residente, en la realidad, las capacidades y actitudes necesarias porque el hospital, con todas las garantías, vea que no las podrá certificar. Como su nombre indica, el residente debe residir en el hospital. Sería ridículo y desnaturalizante seguir llamando 'MIR' a un programa sin la 'I' ni la 'R', y me temo que está empezando a suceder.

Ya finalizada la especialidad, es lógico -aunque en España no ocurre- que los colegios profesionales, si tienen que responsabilizarse de la calidad de sus colegiados, emitan registros de colegiación sólo después de ver y hablar con el nuevo miembro y revisar sus certificados. Esto no es nada difícil ni tampoco un examen, o al menos no debe serlo; constituye un encuentro cordial entre iguales, uno más joven que los demás. En el ámbito estatal o nacional son los denominados Boards en Estados Unidos, propuestos por primera vez en 1908 por el Dr. Derrick Tilton Vail Sr., oftalmólogo, pero no reconocidos hasta 1933 por las entidades Profesionales y estructurados finalmente, en 1970, como American Board of Medical Specialties (ABMS). Los primeros Boards fueron lógicamente los de los fundadores (oftalmología, otorrinolaringología, dermatología y obstetricia-ginecología), y el último, de 1991, el Medical Genetics.

\section{¿Puede la formación adquirirse en cualquier hospital?}

Evidentemente, no. En el hospital, el médico en formación contempla y aprende no sólo el objeto de su especialización, sino un mundo de interacciones interdepartamentales, interper- sonales, medico-administrativas y también de interrelación hospital-sociedad, que tienen que ser correctas y que trascienden la practica de su especialidad. Es una especie de aprendizaje para la convivencia civilizada.

Sin duda, el certificado de acreditación emitido por la Joint Commission International on Accreditation of Hospitals, en España (JCI), es el paso previo ideal para que una facultad de $\mathrm{Me}$ dicina establezca relaciones de cooperación para la formación médica. La JCI es una división de la Joint Commission on Accreditation of Healthcare Organizations.

La acreditación asegura que en un determinado hospital se ejerce una medicina crítica y que su organización provee de transparencia, de un continuo en las responsabilidades y de garantías de estandarización y seguridad. Pero la acreditación no garantiza resultados pormenorizados de alto nivel o excepcionales, simplemente permite una expectativa razonable de que, sin sus imperativos, aquéllos serían prácticamente imposibles. Para que un hospital, además de acreditado, sirva para la enseñanza, hay que analizar sus resultados y estar sometido al denominado peer review, de comparación con centros equivalentes, y aquí ya entran los resultados. Para que un hospital acreditado sirva para la formación de especialistas, además del alto nivel de las especialidades y de pertenecer a lo que llamamos asistencia terciaria, sus profesionales han de disponer de tiempo extra más allá de la función asistencial, y esto, junto a otros factores, tiene un coste que ha de quedar claramente reflejado en el presupuesto de la institución.

Sin embargo, no es necesario ser terciario para obtener el certificado de acreditación, ni el hecho de no tenerlo signifique que no es un buen hospital. Incluso hay hospitales que van más allá, en sus estándares, de los requisitos de la acreditación por razones que ahora no comentaré, básicamente porque la acreditación ha de ser posible para numerosos centros.

Una y otra vez surge el tema de si un centro monográfico, por alto que sea su nivel y sus resultados, debe servir para formar especialistas del tipo médicos residentes. Como es fácil deducir, esos centros de gran prestigio no son completos, no se puede dar en ellos lo que llamamos 'medicina integrativa'. En esos centros no existe, y probablemente tampoco debería existir, el entorno multidisciplinario propio de un hos- 
pital general que crea el ambiente en el cual es deseable que se forme el médico para tener una visión holística del enfermo que trasciende cualquier especialidad. Todo ello sin perjuicio de que en algunos de ellos se realice una determinada técnica mejor que en ninguna otra parte.

El binomio hospital-capacidad para formar médicos es muy estricto y todo incremento cuantitativo ha de acompañarse de una serie de factores, de los cuales el económico es sólo uno. Veamos algunos más:

- El servicio que los acoja debe tener vocación de actuar en esa capacidad docente porque, aunque la formación del residente se produce fundamentalmente por observación-participación en los hechos ('aprender haciendo', como rezaba el lema del John Hopkins), con progresiva asunción de responsabilidades, la supervisión del responsable se mantiene siempre, de forma decreciente, para los actos asistenciales, pero creciente para el resto de la programación. Esto, más la activa participación en las comisiones hospitalarias de autoanálisis y control, la dirección de estudios colaterales, la emisión de informes, etc., exige tiempo.

- El servicio ha de tener casuística suficiente en cantidad y variedad. Lógicamente, el programa debe especificar con exactitud la cantidad de vivencias y el grado progresivo de trabajo independiente a lo largo del período de formación. Esto debe quedar documentado.

- La arquitectura debe facilitar espacios de reflexión y encuentro, además del residencial.

- El archivo central y la biblioteca, y en su caso el uso de las tecnologías de la información y la comunicación, exigen reforzar medios y la accesibilidad a ellos.

- Los servicios centrales (laboratorios, imagen, etc.) siempre se 'recargan' con programas de residentes en marcha. Hay que tenerlo en cuenta y proveer para ello.

- El departamento de contabilidad debe fijar unos costes unitarios por procedimiento, en los que se distinga el coste del programa. Es básico para obtener la financiación de quien corresponda.

Como se ve, el asunto es serio y la simple preparación de los programas puede requerir años. No vale decir: 'como en este hospital practicamos muy buena oftalmología, venga usted y lo convertiremos en oftalmólogo'. Esto era antes, de hecho antes de 1910, cuando Abraham Flexner (1866-1959), tras un largo periplo aprendiendo a enseñar, enseñó a aprender medicina. Había nacido el sistema de formación que aquí llamamos MIR. El periplo había llevado a Flexner de Louisville, en Kentucky, a la Johns Hopkins, a Harvard, a Heidelberg, a la Fundación Carnegie, a analizar 155 escuelas de medicina de Canadá y Estados Unidos a la fundación General Education Board, de Rockefeller: en definitiva, impuso casi nada:

- La articulación de las escuelas médicas con las universidades.

- La exigencia de dos años de ciencias a los aspirantes a ingresar en los estudios de Medicina.

- La integración de la enseñanza práctica en los hospitales.

- La creación de laboratorios y el desarrollo de la investigación.

- La estructuración de las enseñanzas preclínicas y clínicas.

- La dedicación plena y exclusiva para los profesores de enseñanzas preclínicas y clínicas.

- El ajuste entre numero de hospitales y población.

- La normativa sobre las condiciones de admisión, las instalaciones, la calidad de los laboratorios, la competencia del profesorado y los programas.

Para Flexner, 'el mayor coste para un hospital es tener médicos incompetentes'. Y la sociedad creyó en él: obtuvo financiación e inició la medicina moderna que permitió pasar de 'una educación médica del más bajo nivel de ninguna época durante gran parte del siglo XIX en Estados Unidos', como denunció William $\mathrm{H}$. Welch, a la medicina de hoy. La publicación culminante de Flexner fue el informe Medical Education in the United States and Canada, de 1910, que a través del boletín de la Carnegie Foundation se difundió y provocó un alud imparable de adhesiones.

La acción de Flexner y la introducción del concepto de 'hospital standardisation', cuatro años después, por los College of Surgeons de Estados Unidos y Canadá, están en la base de los hospitales como los conocemos hoy. En la estandarización se introdujo la historia clínica, el análisis de la casuística, el decir y dejar documentada la verdad de lo que sucede con los pacientes, la estadística... Visto el origen de los errores y trazada la responsabilidad, se corrigen, 
y si siguen, se recomienda el cese del responsable 'incorregible'. La estandarización afecta a todo, procedimientos, equipo, etc. Nace el derecho universal a obtener todos lo mejor. Pues bien, transcurridos 100 años, el profesor Ian Gilmore, presidente del Royal College of Physicians británico, nos tiene que recordar: 'The biggest single factor in delivering high quality safe healthcare, is the timely availability of accurate and relevant information about the patient'. Y aunque Gilmore se refiere al registro y transmisión electrónica de la historia clínica, el concepto es el mismo: $a c c u-$ rate and relevant, 'precisa y significativa'.

He querido recordar la acreditación y la estandarización como pilares básicos de la buena práctica en los centros asistenciales en general $\mathrm{y}$, particularmente, en aquellos donde aprender medicina. Sólo quisiera pedir unos momentos de reflexión a cualquiera que trabaje en un hospital, en cualquier nivel: que vea si en su hospital se cumplen esos requisitos, casi cien años después de establecidos. Luego pediré esta misma reflexión para los responsables extrahospitalarios. La medicina actual enfrenta a nuestros hospitales, cada día, a nuevos procedimiento y técnicas que requieren la aplicación exacta de los contenidos de aquellas normativas, pero la rutina, las prisas y un largo etcétera hacen que se vayan descuidando. Un nuevo procedimiento debería acompañarse de sus correspondientes instrucciones permanentes y garantía de su aprendizaje, protocolos escritos y rastros que permitan su auditoría, y no siempre es así. De los cerca de 1.000 hospitales que existen en España, sólo cinco disponen de un certificado de acreditación emitido por la JCI y la estandarización no existe. La única explicación lógica es que estos sistemas, antes de solicitar la inspección por la entidad independiente correspondiente, presuponen una autoevaluación para poner la casa en orden y siempre se llega a lo mismo: hay que tener voluntad, presupuesto y disciplina. Sin esa tríada, nada es posible. Uno siempre piensa lo mismo: si con todo, nuestros hospitales, gracias al sistema MIR, presentan niveles medios bastante buenos, qué no sería con la tríada plenamente en vigor.

La medicina avanza a gran velocidad; las tecnologías de la información y la comunicación hacen que los 3-5 años que tardaba el mundo médico hace sólo 50 años en enterarse de una novedad significativa para poder aplicarla, se hayan reducido, afortunadamente, a días. Los médicos que hayan aprendido a aprender (uno de los objetivos del Espacio Europeo de Educación Superior) se adaptarán en tiempo útil y sabrán distinguir lo significativo de lo superfluo. Es más dudoso que las organizaciones que proveen soporte al proceso funcional y educacional de los hospitales sean capaces de hacerlo; para ellas no existe un plan de educación continuada. Eso producirá desajustes graves. De hecho, ya se producen. La misma estructura física y organizativa de los hospitales, la práctica, sufrirá grandes cambios en poco tiempo. Sólo unas palabras sueltas para entender esto: genética, biología molecular, proteómica, farmacogenómica y farmacogenética, robótica, nanotecnología, neuroquímica, trasplantes celulares, bioinformática... La frase 'from lab to clinic' expresa cuán rápidamente se trasladan los frutos de la investigación a la práctica asistencial y cuán alerta deben estar las organizaciones asistenciales para adaptarse a esto si no quieren ser arrolladas. Y cuán formados deben estar los elementos humanos para no ser, ellos mismos, arrollados por la 'novedad', considerando que:

- El manejo de técnicas cuyas entrañas desconoce lo llevan a ser un mero aplicador. Sólo puede evaluarlas por el resultado, pero no puede modificar instrumentos que han creado otros, procedentes de disciplinas lejanas, para las cuales no domina el lenguaje, ni intuye aún el riesgo de que la robótica lo aparte del circuito terapéutico, del mismo modo en que otras técnicas lo van apartando del proceso diagnóstico.

- La potencia técnica y económica de la industria, que hace tiempo que ha visto que en la salud hay un filón inacabable que puede manejar a voluntad. De hecho, es la primera en uso y empleo de recursos, con tendencia al alza. La industria se emplea en modelar el consumo, y además de ser un potente motor de avances incuestionables, también produce un porcentaje que no cuantificaré, pero que es muy alto, de auténtico junk. A los valores positivos, sólo ella puede llegar. Piénsese en el coste de producir una molécula original terapéuticamente eficaz.

No hay duda de que algunos cerebros con simple sentido común, después de estudiar y recordar las enseñanzas de Flexner, y refrescar la estandarización y la acreditación, deberían, sin saltarse ninguno de aquellos preceptos, aconsejar 
la actualización de las estructuras a las nuevas funciones y a las aspiraciones de los seres humanos que desean realizarse médicamente. Alguna Carnegie española debería financiarlo, y las hay, aunque no parece que estén por la labor. Y básicamente, quienes tengan entre sus funciones el servicio público, deberían asumir esos consejos. La sociedad se lo demandará en un feedback más o menos lento, según sea el progreso cultural y educacional que, al compás de la directiva de Bolonia, será explosivo.

Con los últimos párrafos llego al final y quizás al objetivo de este artículo:

- Recordar que los grandes avances en el progreso de la enseñanza o asistencia médica arrancan del esfuerzo de un hombre o grupo, que lanza unas ideas que permean la sociedad y se imponen (Flexner, los Colegios de Cirujanos de América y Canadá).

- La cadena de responsabilidades no admite disimulos ni fracturas si el ser humano individual, que ha de recibir el beneficio, cuenta.

- No cabe resistirse porque en la base está la comunidad, que acaba comprendiéndolo y lo exige porque, en realidad, en ella reside el poder.
Todo ello significa:

- Los responsables de hospitales de titularidad pública o privada y entornos extrahospitalarios deben cumplir con su deber. Los primeros son entes entre el hospital mismo y la sociedad. Aquellos que tienen poder de decisión sobre el hospital en lo financiero y en muchos otros aspectos, bien harían en estar al día de los conceptos evocados e incorporarlos a sus preocupaciones. Los segundos, los entornos, los medios, que etiquetan e interpretan, pueden hacer un gran bien o un gran mal y sus verdades ayudan mucho.

- Los agentes activos (directores de hospital y de servicio, enfermeras, técnicos, administrativos, profesores, y resto del personal) deben sentirse en todo momento seguros en su responsabilidad y en su quehacer, y parte esencial de esa seguridad reside en saber que su soporte emana del más alto nivel administrativo o político, pero esencialmente del sentimiento que dejen en el sujeto pasivo de todo el sistema: los usuarios.

- Cualquier quiebra o incultura pone en peligro el sistema. 